


\title{
Nuclear Inositide Signaling Via Phospholipase C
}

\author{
Stefano Ratti, ${ }^{1}$ Sara Mongiorgi, ${ }^{1}$ Giulia Ramazzotti, ${ }^{1}$ Matilde Y. Follo, ${ }^{1}$ \\ Giulia A. Mariani, ${ }^{1}$ Pann-Ghill Suh, ${ }^{2}$ James A. McCubrey, ${ }^{3}$ Lucio Cocco, ${ }^{1 *}$ \\ and Lucia Manzoli ${ }^{1}$ \\ ${ }^{1}$ Cellular Signalling Laboratory, Department of Biomedical and Neuromotor Sciences, University of Bologna, via \\ Irnerio 48, 40126 Bologna, Italy \\ ${ }^{2}$ School of Life Sciences, Ulsan National Institute of Science and Technology, Ulsan, Republic of Korea \\ ${ }^{3}$ Department of Microbiology and Immunology, Brody School of Medicine, East Carolina University, Greenville \\ 27834, North Carolina
}

\begin{abstract}
The existence of an independent nuclear inositide pathway distinct from the cytoplasmic one has been demonstrated in different physiological systems and in diseases. In this prospect we analyze the role of PI-PLC $\beta 1$ nuclear isoform in relation to the cell cycle regulation, the cell differentiation, and different physiopathological pathways focusing on the importance of the nuclear localization from both molecular and clinical point of view. PI-PLC $\beta 1$ is essential for G1/S transition through DAG and Cyclin D3 and plays also a central role in G2/M progression through Cyclin B1 and PKC $\alpha$. In the differentiation process of C2C12 cells PI-PLC $\beta 1$ increases in both myogenic differentiation and osteogenic differentiation. PI-PLC $\beta 1$ and Cyclin D3 reduction has been observed in Myotonic Dystrophy (DM) suggesting a pivotal role of these enzymes in DM physiopathology. PI-PLC $\beta 1$ is also involved in adipogenesis through a double phase mechanism. Moreover, PI-PLC $\beta 1$ plays a key role in the normal hematopoietic differentiation where it seems to decrease in erythroid differentiation and increase in myeloid differentiation. In Myelodysplastic Syndromes (MDS) PI-PLC $\beta 1$ has a genetic and epigenetic relevance and it is related to MDS patients' risk of Acute Myeloid Leukemia (AML) evolution. In MDS patients PI-PLC $\beta 1$ seems to be also a therapeutic predictive outcome marker. In the central nervous system, PI-PLC $\beta 1$ seems to be involved in different pathways in both brain cortex development and synaptic plasticity related to different diseases. Another PI-PLC isozyme that could be related to nuclear activities

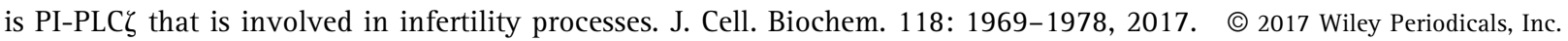

KEY WORDS: SIGNALING; INOSITIDES; NUCLEUS; PHOSPHOLIPASE C; PATHOPHYSIOLOGY

\section{NUCLEAR INOSITIDE SIGNALING}

During the last 30 years nuclear inositide signaling has been investigated in many different systems and diseases. The eukaryotic outer nuclear membrane is made of protein and phospholipids and comes from the endoplasmic reticulum. For this reason cell fractionation showed nuclei with a high amount of lipids, due to the incomplete removal of the nuclear double layer. After the total removal of the nuclear envelope by detergents it was interesting to observe that still a significant quantity of lipids was present inside the nucleus. Even though nuclear Phosphatidylcholine (PC) is the main phospholipid, Phosphatidylinositols (PIs), present in small amount [Tribble et al., 2016], have specific role in intranuclear signaling. PIs play an important role in nuclear function regulation and behave differently from their counterparts in the cytoplasm. The autonomous nuclear PI cycle in eukaryotic cells is involved in different regulation processes, from cell proliferation to differentiation and many others [Irvine, 2003]. In this prospect we will try to describe the state of the art of nuclear inositide signaling via Phospolipase C (PI-PLC), the pathophysiological involvement of PIPLCs, the fields remained undiscovered, and the possible researches related to this intriguing and complicated world.

\section{HISTORICAL BACKGROUND}

In 1987, for the first time, using Friend cell highly purified nuclei (washed free of nuclear membrane by Triton) Cocco et al. suggested that Phosphatydilinostil-4-phosphate (PI4P) and Phosphatydilinostil-4,5-biphosphate $\left(\mathrm{PI}(4,5) \mathrm{P}_{2}\right)$ were specifically present inside the

Grant sponsor: Intesa San Paolo; Grant sponsor: Italian MIUR-PRIN 2015; Grant sponsor: East Carolina University; Grant number: \#111104; Grant sponsor: National Research foundation of Korea.

${ }^{*}$ Correspondence to: Lucio Cocco, Cellular Signalling Laboratory, Department of Biomedical and Neuromotor Sciences, University of Bologna, via Irnerio 48, 40126 Bologna, Italy. E-mail: lucio.cocco@unibo.it

Manuscript Received: 17 January 2017; Manuscript Accepted: 18 January 2017

Accepted manuscript online in Wiley Online Library (wileyonlinelibrary.com): 20 January 2017

DOI 10.1002/jcb.25894 • (C) 2017 Wiley Periodicals, Inc. 
nucleus. This work proposed that these PIs could have an important role in signal transduction parallel to the more documented cytoplasmic one [Cocco et al., 1987]. Later, the stimulation with Insulin-like growth factor-1 (IGF-1) of Swiss 3T3 fibroblasts demonstrated the IGF-1 regulation of a distinct nuclear PI metabolism [Cocco et al., 1988]. Moreover, the treatment of Swiss 3T3 fibroblasts with IGF-1 determined a decrease in nuclear PI4P and $\mathrm{PI}(4,5) \mathrm{P}_{2}$ with a consequential increase in Diacylglycerol (DAG) and therefore a translocation of protein kinase $\mathrm{C}$ (PKC) inside the nucleus. On the contrary Swiss 3T3 fibroblast treated with bombesin showed activation only of the classical cytoplasmic pathway [Divecha et al., 1991; Martelli et al., 1991]. The presence of these enzymes at the nuclear level suggests their essential role in nuclear signaling that, otherwise, would not be possible to be realized at the cytoplasmic level only. Moreover, for being able to identify a certain amount of these PIs inside the nucleus their presence should be stable or, at least, long enough to be localized and of course to perform a certain nuclear activity. For these reasons these data opened a new field of research in nuclear inositide signaling via PI-PLC, independent from the cytoplasmic one. Since the beginning of these experiments up to now there have been lots of enlightenments on this theme, but many aspects remain still unclear and critical.

\section{NUCLEAR INOSITIDE SUBCELLULAR LOCALIZATION AND FUNCTIONS}

If it is known that PIs are also present within the nucleus, their precise topography is still under investigation. Different studies focused on nuclear compartments in order to clarify PI nuclear geography. The main difficulty is that some PIs have different possible routes of synthesis and also removal that are localized in different regions inside and outside the nucleus [Irvine, 2003]. For example $\mathrm{PI}(4,5) \mathrm{P}_{2}$ could be synthetized by PIP-5-kinase (PIP5K) or by PIP-4-kinase (PIP4K) starting from different substrates, respectively PI-4-phosphate(PI-4P) and PI-5P [Bulley et al., 2015]. PIP5K and PIP4K have both different isoforms with different localization from the plasma membrane to the nucleus itself: PIP5K $\alpha$ (plasma membrane), PIP5K $\beta$ (both plasma membrane and perinuclear region), and PIP5K $\gamma$ (in focal adhesions and adherent junctions in epithelial cells); PIP4K2A (plasma membrane and cytoplasm), PIP4K2B (nucleus), and PIP4K2C (endomembrane compartment) [Bultsma et al., 2010]. Considering our geographical focus the main question here is which class of these two PIPKs is responsible for the $\mathrm{PIP}(4,5) \mathrm{P}_{2}$ synthesis inside the nucleus. Several experiments evidenced that PIP5K was accountable of almost $90 \%$ of $\mathrm{PI}(4,5) \mathrm{P}_{2}$ production within the nucleus, while PIP4K was responsible for only a little amount [Balla and Balla, 2006]. These data suggest that there are several possible pathways for the same PI synthesis, related to its localization and function. Moreover, PI4-kinase is mainly present in nuclear peripheral matrix, instead PI-PLC, DAG kinase, and PI4P5kinase seem to be present in the internal one [Payrastre et al., 1992]. On rat liver cells the isolation of nuclei with Triton X-100 at a concentration of $0,04 \%$, in order to remove completely the bilayer nuclear envelope, showed that there were still several nuclear pools of different PIs such as PI, PI(4,5) $\mathrm{P}_{2}$, phosphatidic acid (PA), DAG, and phosphatidylinositol 3,4,5-triphosphate $\left(\mathrm{PI}(3,4,5) \mathrm{P}_{3}\right)$ [Vann et al., 1997]. Moreover, it has been evidenced that $\operatorname{PI}(4,5) \mathrm{P}_{2}$ is related to nuclear speckles. These speckles are interchromatin granule clusters where different PIs and other related enzymes are localized regulating chromatin remodeling, nuclear functions and RNA splicing [Boronenkov et al., 1998; Osborne et al., 2001].

The complexity of this topic is related to the difficulties of reproducible nuclear purification techniques and the variables in the metabolism and localization of these enzymes. In any case, these data suggest that PIs are somehow and at some point present inside specific nuclear regions and can therefore perform nuclear functions. Their temporary or stable permanence within the nucleus should then be useful for some nuclear activities that, instead, would not be possible in the cytoplasm [Tribble et al., 2016].

Indeed nuclear PIs are involved in many activities and can control several fundamental nuclear processes. As we saw above, their localization in specific nuclear regions named speckles strongly suggests their role in transcription regulation and in chromatin remodeling [Boronenkov et al., 1998]. Proteins can bind PIs through Plant Homeo Domains (PHD) fingers making the interaction with other nuclear proteins, ligands, histones, and chromatin possible. PIs can modify histone N-tails characteristics regulating the activity of several enzymes involved in chromatin remodeling, leading to different chromatin architecture and therefore gene transcription. Moreover, they are able to modulate gene transcription interacting with transcription complexes and nuclear proteins. PIs can also directly affect RNA maturation affecting several gene expression and therefore PIs can have pivotal roles in different physiopathological processes [Shah et al., 2013]. Among the PI metabolizing enzymes that are present in the nucleus of eukaryotic cells we will mainly focus on the PI-PLCs and its related enzymes trying to analyze their physiopathological role.

\section{NUCLEAR PI-PLC FEATURES}

PI-PLCs are a class of 13 isozymes, divided in six families: PI-PLC- $\beta$ (1-4), $-\gamma(1-2),-\delta(1,3$, and 4$),-\varepsilon,-\zeta$, and $\eta(1-2)$ [Gresset et al., 2012]. These different enzymes share conserved structural features and can all hydrolyze $\mathrm{PI}(4,5) \mathrm{P}_{2}$ in response to several ligand bonds with cell surface receptors. This process produces DAG and inositol-1,4,5trisphosphate $\left(\mathrm{IP}_{3}\right) \cdot \mathrm{IP}_{3}$ is responsible for the release of $\mathrm{Ca}^{2+}$ that, together with DAG, leads to the activation of PKC. This molecular cascade is essential for transmembrane signal transductions that result in cellular communication, proliferation and differentiation. Beyond these routes, PI-PLCs are also localized within the nucleus and are independent from the cytoplasmic pathways. As seen before, we now know that several PIs are localized inside the nucleus itself, but the specific mechanism of activation is still uncertain. Cytoplasmic PIPLC activation is related to G-protein-coupled receptor (GPCR) and receptor tyrosine kinase (RTK), but the nuclear activation apparatus is not completely defined. It is possible that IGF-I dependent signal, through a negative feedback by PKC $\alpha$, modulates nuclear PI-PLC activation [Xu et al., 2001]. Therefore, it seems that the nuclear route has different initial triggers and different mechanism compared to the cytoplasmic ones. Between the different PI-PLC isozymes we will 
focus on PI-PLC $\beta 1$, the most investigated PI-PLC within the nucleus [Martelli et al., 1992].

PI-PLC $\beta 1$ gene is located on the short arm of chromosome 20 (20p12) and produces two splicing variants: PI-PLC $\beta 1 \mathrm{a}(150 \mathrm{kDa})$ and PI-PLC $\beta 1 \mathrm{~b}(140 \mathrm{kDa})$ that differentiate one another for the Cterminal sequence and their localization [Bahk et al., 1998]. Specifically, both of them have a Nuclear Localization Sequence (NLS), but PI-PLC $\beta 1$ a also has a Nuclear Export Sequence (NES), and for this reason it localizes also in the cytoplasm [Follo et al., 2006]. On the other hand, PI-PLC $\beta 1 \mathrm{~b}$ is mainly located inside the nucleus [Martelli et al., 2005]. In order to clarify the mechanism of PI-PLC $\beta 1$ nuclear import, PI-PLC $\beta 1$ a isoform was mutated at the C-terminal sequence. This experiment resulted in a significant reduction in the nuclear localization of the enzyme confirming that the C-terminal sequence is involved in the nuclear import [Kim et al., 1996].

\section{NUCLEAR INOSITIDE SIGNALING IN CELL CYCLE REGULATION}

We will now analyze the role of this enzyme in cell cycle regulation even if we have to consider the difficulties of synchronizing or arresting in a specific cell cycle phase our cell population. Moreover, working with lipids makes always hard to obtain highly purified nuclei in order to be sure to observe the nuclear PI pools.

\section{NUCLEAR PI-PLC $\beta 1$}

Nuclear PI-PLC $\beta 1$ is involved in cell cycle progression and can regulate the levels of different cyclins such as Cyclin D3, Cyclin E, and Cyclin B1. Cells overexpressing PI-PLC $\beta 1$ show an increase of Cyclin D3 levels and a higher percentage of cells in $S$ phase, compared to the controls in K562 human erythroleukemia cell line [Poli et al., 2016a]. Moreover, PI-PLC $\beta 1$ can regulate Cyclin E that plays a pivotal role in S-phase progression [Piazzi et al., 2015]. PIPLC $\beta 1$ can therefore modulate G1/S cell cycle transition through these cyclins. Another class of nuclear PIs that very recently resulted to be essential during this cell cycle phase in K562 cells are the Diacylglycerol Kinases (DGKs) and particularly the DGK $\alpha$ [Poli et al., 2016b]. Furthermore, it is interesting to observe that PI-PLC $\beta 1$ can be also involved in G2/M cell cycle progression. In 2005 LukinovicSkudar et al. [2005] described two picks of PI-PLC during both G1/S transition and G2/M progression. We will now analyze the nuclear PI-PLC $\beta 1$ mainly related PIs.

\section{NUCLEAR DAG}

As it occurs in the cytoplasm, also in the nucleus PI-PLC activity is responsible for DAG and IP3 synthesis. IP3 should perform his activity as a $\mathrm{Ca}^{2+}$ mobilizer inside the nucleus, too and nuclear DAG shows level changes during the cell cycle. As before, it seems that DAG follows nuclear PI-PLC oscillations observed in G1/S and G2/M phases [Lukinovic-Skudar et al., 2005]. These data gave more strength to the previous experiments performed on HL60 cell lines [Sun et al., 1997] and U937 cell lines [Deacon et al., 2002] underlining the pivotal role of PI-PLC $\beta 1$ and its related enzymes in cell cycle regulation. It has been demonstrated a peak in nuclear DAG production followed by an increase of PKC $\alpha$ and cyclin B1 in G2/M
K562 synchronized cells. Indeed the inhibition of PI-PLC activity leads to a reduction in nuclear $\mathrm{IP}_{3}$ synthesis with a consequential decrease in nuclear translocation of PKC $\alpha$ and Cyclin B1 that are essential in G2/M phase [Poli et al., 2014]. As previously shown the role of PI-PLC is central for the PI cascade in cell cycle regulation and interaction with Cyclins. The probable consequence of DAG generation within the nucleus is an activation of nuclear PKC that leads to several cellular processes.

\section{NUCLEAR PKC}

PKC can be present inside the nucleus in different isoforms [Irvine, 2003]. Both its two isoforms PKC $\alpha$ and PKC $\beta I I$ can translocate inside the nucleus after DAG stimulation. As for other PIs, this translocation process should be long enough to be observed and to be able to play some role in nuclear pathways. In Swiss 3T3 cell line PKC $\alpha$ can translocate inside the nuclei as a result of a probable nuclear DAG stimuli [Martelli et al., 1991] and also in U937 cell line the translocation of PKC $\beta$ II inside the nuclei was showed after DAG increase [Deacon et al., 2002]. The intriguing data is that PKC can translocate inside the nuclei also after other stimuli such as IGF-1, Platlet derived growth factor or Bryostatin [Irvine, 2003]. In addition to these data the same stimuli can determine the translocation in the nucleus of both PKC $\alpha$ and PKC $\beta$ II isoforms. Another interesting aspect is that a PI-PLC $\beta 1$ stable overexpression is related with a decrease of PKC $\alpha$ levels, and this decrease is possibly related to PKC $\alpha$ hyper- and stable activation induced by DAG continuous production [Poli et al., 2014]. In G2/M-synchronized MEL cells the change in levels of PI-PLC $\beta 1$ altered nuclear PKC $\alpha$ accumulation with consecutive alteration in the mitotic process [Fiume et al., 2009]. PKC has indeed different targets, like the Lamin B1, strongly linked to the cellular cycle. As we saw above, there is a PI cascade that starting from nuclear PI-PLC $\beta 1$, through DAG and $\mathrm{IP}_{3}$ or and $\mathrm{Ca}^{2+}$, activates PKC $\alpha / \mathrm{PKC} \beta \mathrm{II}$ and other molecules controlling the cell cycle. It is possible that DAG and so PKC regulate cell cycle and cell proliferation in relation to different signals and cell population.

\section{NUCLEAR PI(4,5) $\mathrm{P}_{2}$}

$\mathrm{PI}(4,5) \mathrm{P}_{2}$ is not only a substrate for PI-PLC, but this PI seems to be essential in cell cycle progression and other nuclear functions. As reported before, $\mathrm{PI}(4,5) \mathrm{P}_{2}$ is largely produced by PIP5K that can interact with Retinoblastoma protein $(\mathrm{pRb})$ which, in turn, controls the progression to $S$ phase in the cell cycle [Divecha et al., 2002]. The link between PIP5K and pRb can determine PIP5K activation and then $\mathrm{PI}(4,5) \mathrm{P}_{2}$ synthesis. For this reason $\mathrm{PI}(4,5) \mathrm{P}_{2}$ plays an important role in cell cycle progression and in the nuclear PI-PLC cascade. Moreover, $\mathrm{PI}(4,5) \mathrm{P}_{2}$ could have structural and also regulatory function in RNA splicing, indeed this class of PIs resides in the interchromatin granular structures interacting dynamically with different chromatin structures during the cell cycle [Osborne et al., 2001] (Fig. 1).

\section{NUCLEAR PI-PLC $\beta 1$ IN CELL DIFFERENTIATION}

Nuclear PI-PLC $\beta 1$ plays a crucial role in cell differentiation of different cell systems. We will focus on the hematopoietic, 


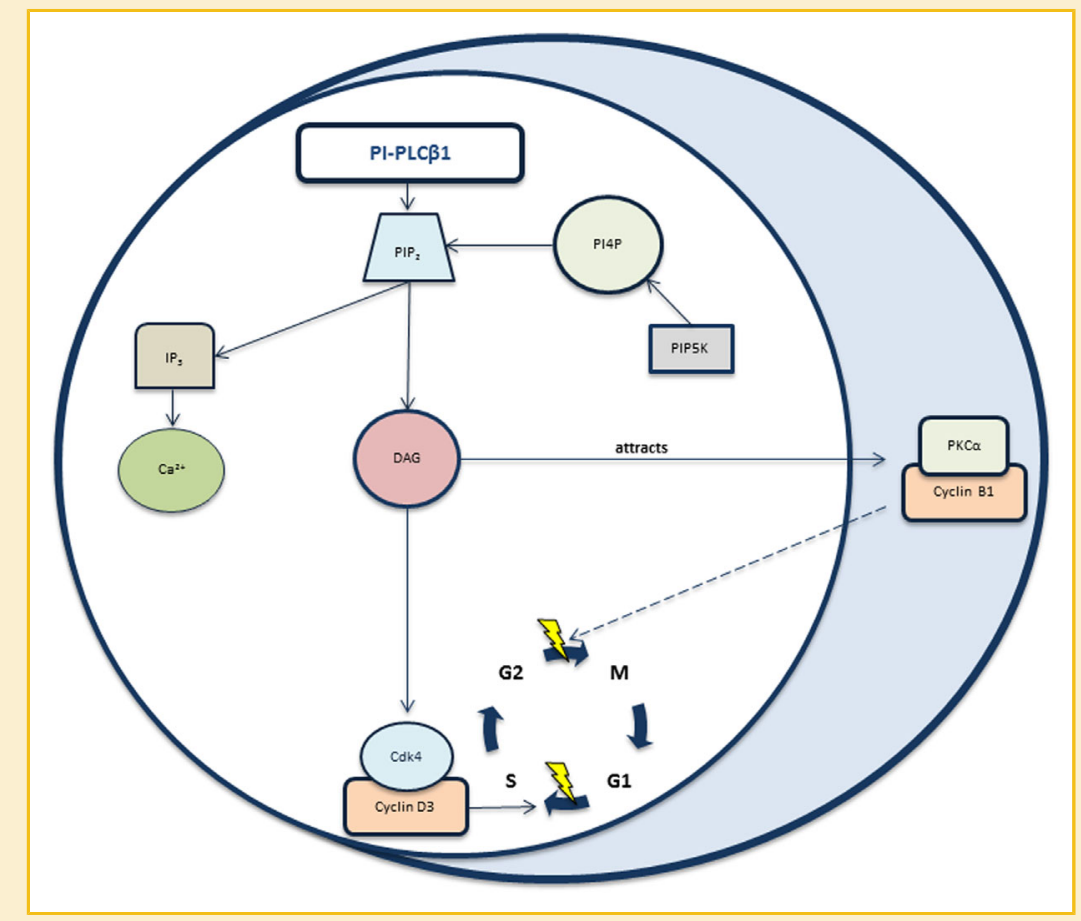

Fig. 1. In this figure, we can observe the nuclear PI-PLC $\beta 1$ pathway related to the cell cycle. PI-PLC $\beta 1$ is essential for G1/S transition through DAG, Cyclin D3-Cdk4 complex, and plays a central role in G2/M progression through Cyclin B1 and PKC $\alpha$. Indeed DAG attracts Cyclin B1 and PKC $\alpha$ from the cytoplasm into the nucleus. We can also see that PIP5K is pivotal for $\mathrm{PI}(4,5) \mathrm{P} 2$ production within the nucleus, while PIP4K was responsible for only a little amount.

osteogenic, myogenic, and adipogenic systems in order to concentrate on the different function and variations of this pivotal PI in cell differentiation. Indeed PI-PLC $\beta 1$ seems to have reverse modulation in distinctive cell systems (Fig. 2).

\section{HEMATOPOIETIC DIFFERENTIATION}

PI-PLC $\beta 1$ has shown to be involved in the hematopoietic system performing pivotal roles in both erythroid and myeloid differentiation. Interestingly this nuclear PI has opposite behavior in these two subsystems: in the erythroid differentiation PI-PLC $\beta 1$ seems to decrease, whereas in the myeloid differentiation PI-PLC $\beta 1$ appears to increase [Follo et al., 2015]. More specifically in murine erythroleukemia cells (MEL) and in normal CD34+ cells with erythropoietin stimulation toward erythroid lineage PI-PLC $\beta 1$ has shown to decrease during the erythroid differentiation process. Furthermore, PI-PLC $\beta 1$ has presented the same trend also in Myelodysplastic Syndromes (MDS), responding to erythropoiesis stimulating agents [Follo et al., 2012]. These data suggest that PIPLC $\beta 1$ plays an inhibiting role in erythroid differentiation that is countered by PI-PLC $\gamma 1$, another PI-PLC isozyme that increases in erythroid differentiation. The negative role of PI-PLC $\beta 1$ in erythroid differentiation was first observed inside the nucleus with an increase of $\mathrm{PI}(4,5) \mathrm{P}_{2}$ and a decrease of both PI-PLC $\beta 1$ and DAG in differentiating MEL cells [Cocco et al., 1987].

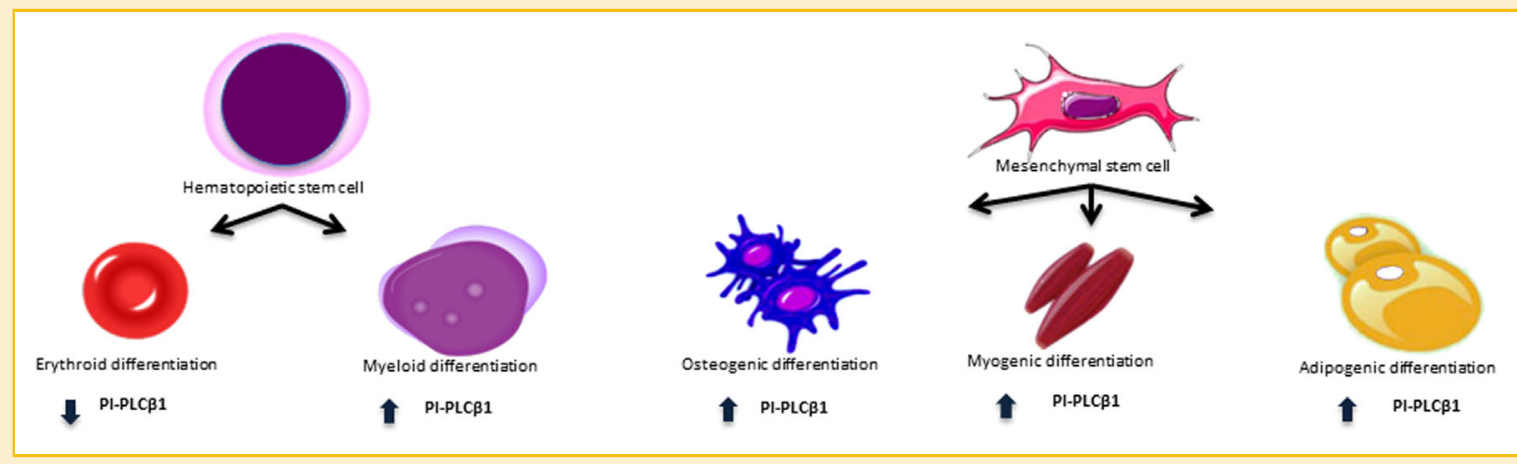

Fig. 2. PI-PLC $\beta 1$ level changes during cell differentiation. PI-PLC 1 increases during the normal osteogenic and myogenic differentiation in C2C12 cells. PI-PLC $\beta 1$ also increases in adipogenic differentiation in 3T3-L1 cells. During erythroid differentiation there is a decrease in PI-PLC $\beta 1$ level demonstrated in MEL, CD34+, and MDS cells. PIPLC $\beta 1$ seems to increase in MDS cells and also in MDS patients samples during the myeloid differentiation induced by azacitidine. 
In myeloid differentiation the role of nuclear PI-PLC $\beta 1$, studied using a MDS experimental model, seems to be opposite to the one just observed in erythroid differentiation. Indeed MDS cells treated with azacitidine, a hypomethylating agent used for the induction of a normal myeloid differentiation, determined a decrease of PI-PLC $\beta 1$ promoter methylation and thus an increase of nuclear PI-PLC $\beta 1$. The potential positive role of PI-PLC $\beta 1$ in myeloid differentiation could be correlated with the recruitment of specific myeloid transcription factors, such as Myeloid zinc finger-1 (MZF-1), during PI-PLC $\beta 1$ expression increase [Cocco et al., 2016]. Observing also the clinical aspects of MDS patients treated with azacitidine it is very interesting to point that the increase of PI-PLC $\beta 1$ expression is not only related to a favorable clinical response, but also to the durable response to the treatment. Indeed the reduction in PI-PLC $\beta 1$ expression can be associated with a loss of response even in patients that had, at first, a positive response to AZA [Filì et al., 2013]. These data about the variation in PI-PLC $\beta 1$ expression could be useful to divide MDS patients that could undergo to AZA treatment into two groups: the ones who will probably show a favorable long-term response to the drug and the ones who would be refractory to the treatment and thus could avoid its intrinsic risks [Cocco et al., 2015]. Afterwards, we will see MDS clinical aspects and possibilities related to PI-PLC $\beta 1$ and other PIs more in detail.

\section{OSTEOGENIC DIFFERENTIATION}

Nuclear PI-PLC $\beta 1$ seems to be involved also in osteogenic differentiation in relation to different osteogenic molecules. The osteogenic differentiation is stimulated by several signaling pathways, mainly controlled by bone morphogenic proteins (BMPs), Osterix (0sx/Sp7), and Runt-related transcription factor 2 (Runx2).

C2C12 cells, a mouse skeletal muscle cell line, can differentiate into osteoblasts after BMP-2 stimulation [Katagiri et al., 1994]. Very interestingly, during the osteogenesis induced by BMP-2 in C2C12 cells it has been shown an increase of PI-PLC $\beta 1$ expression. In addition the down-regulation of PI-PLC $\beta 1$ gene expression results in an inhibition of the osteogenic differentiation process induced in C2C12 by BMP-2, as demonstrated by Alkaline phosphatase (ALP) reduction. Instead PI-PLC $\beta 1$ overexpression determines an increase of osteogenic differentiation as demonstrated by the rise of ALP and Osx/Sp7 [Ramazzotti et al., 2016]. Indeed ALP and Bone gammacarboxyglutamic acid containing protein (Bglap or Osteocalcin) are osteoblast marker genes that increase in response to the action of the main bone morphogenic proteins that control the osteoblastic differentiation.

PI-PLC $\beta 1$ is also related to miR-124, another molecule that is very relevant during the control of osteogenic differentiation. miR-124 can promote the myogenic differentiation and inhibit the osteogenic differentiation by regulating 0sx/Sp7. Specifically the inhibition of miR-214 in C2C12 cells leads to an increase of PI-PLC $\beta 1$ protein level and furthers the osteogenesis [Feng et al., 2011]. Another very intriguing aspect of the relation between nuclear PIs and osteogenic differentiation is that, even without BMP-2, the overexpression of PI-PLC $\beta 1$ still determines a certain level of osteoblast differentiation, albeit reduced. This could signify the capability of PI-PLC $\beta 1$, per se, to induce osteogenic differentiation through possible downstream molecules.

\section{MYOGENIC DIFFERENTIATION}

There are different pathways related to the myogenic differentiation that can be activated by several events and that can lead to various cellular processes. The two main families of the transcription factors related to myogenic differentiation are the Myogenic Regulatory Factors (MRFs) and the Myocyte Enhancer Facotr-2 (MEF2) [Ramazzotti et al., 2016]. C2C12 cells under low serum conditions can differentiate into myotubes. These cells synthesize muscle specific proteins like MyoD and Myogenin that are essential for the myoblast alignment, elongation and fusion that allows myotube formation. During the myotube formation nuclear PI-PLC $\beta 1$ increased in $\mathrm{C} 2 \mathrm{C} 12$ cells induced to differentiate. This PI-PLC $\beta 1$ increase was associated with a Cyclin D3 level increase, too [Cocco et al., 2016]. MyoD stimulates Cyclin D3 expression that can thus binds the unphosphorylated $\mathrm{pRb}$ responsible for the myoblast cell cycle recession [Ramazzotti et al., 2016]. Moreover, Cyclin D3 promoter has a region activated by PI-PLC $\beta 1$, and this could be responsible for Cyclin D3 accumulation in relation to PI-PLC $\beta 1$ activity [Faenza et al., 2012]. More specifically the transcription factor c-jun needs to bind to Cyclin D3 promoter in order to determine its activation during PI-PLC $\beta 1$ signaling cascade [Ramazzotti et al., 2016]. This possible pathway starts with PI-PLC $\beta 1$ catalytic activity that leads to $\mathrm{IP}_{3}$ synthesis, which is phosphorylated by Inositol Polyphosphate Multikinase (IPMK) with the production of higher phosphorylated inositol species such as IP4, IP5, and IP6. IP5 can induce $\beta$-catenin nuclear translocation, activating c-jun, thus Cyclin D3 promoter. $\beta$-catenin target genes are involved in several cellular processes like proliferation, differentiation, and cancerogenic stimuli. This pathway could explain the key role of nuclear PI-PLC $\beta 1$ in myogenic differentiation. It is worthwhile to remember that also PI-PLC $\gamma 1$ expression increases during the myogenic differentiation. Interestingly, even if Cyclin D3 is essential in myogenic differentiation it seems not to be involved during osteogenesis. Indeed Cyclin D3 levels do not increase during osteogenesis neither after PI-PLC $\beta 1$ overexpression, for this reason it seems to promote myogenic, but not osteogenic differentiation [Ramazzotti et al., 2016]. We will analyze later in this prospect the clinical correlation between PI-PLC $\beta 1$ and myogenic differentiation in the Myotonic dystrophies.

\section{ADIPOGENIC DIFFERENTIATION}

Nuclear PI-PLC $\beta 1$ and Cyclin D3 could be involved in adipocyte differentiation, too. During the differentiation of 3T3-L1 adipocytes there is an up-regulation of the nuclear PI-PLC $\beta 1$. Interestingly there have been observed two different phases of this differentiation process. In the first phase, after $5 \mathrm{~min}$ of incubation with the differentiation media, the pathway is regulated by $\operatorname{PKC} \alpha$ pathways. In this phase no further nuclear PI-PLC $\beta 1$ recruiting is needed, probably because the nuclear PI-PLC $\beta 1$ levels are already sufficient at this stage. In the second phase, after 2 days of differentiation, there is the necessity of PI-PLC $\beta 1$ nuclear translocation, independently from PKC $\alpha$ control. Interestingly the overexpression of PI-PLC mutants, lacking the nuclear localization sequence showed that both PI-PLC $\beta 1$ phases are essential for an effective differentiation. Moreover, the inhibition of PI-PLC $\beta 1$ avoided the overexpression of the complex formed by Cyclin D3 and Cyclin-dependent-kinase-4 
(CDK4), suggesting a role of PI-PLC $\beta 1$ in the cell cycle control during adipogenic differentiation [O'Carroll et al., 2009].

\section{NUCLEAR PI-PLCS IN DISEASES}

PI-PLCs have been studied as pivotal enzymes, clinical molecular targets, and clinical prognostic/diagnostic factors in many physiopathological processes. The different PI-PLC isozymes are related to several pathologies in many human tissues and the understanding of these mechanisms could open new path for developing innovative therapeutic strategies. In this prospect we will focus on the diseases characterized by alterations of nuclear PI-PLCs.

\section{MYELODYSPLASTIC SYNDROMES (MDS)}

MDS are a heterogeneous group of pathologies characterized by hematopoietic stem cell alteration that lead to anemia, neutropenia, bleeding and infections. Moreover, 30\% of MDS patients can evolve into acute myeloid leukemia (AML). The IPSS (International Prognostic Scoring Systems) and the WPSS (WHO classificationbased Scoring System) classifications, mainly based on the blast number and karyotype, are used to divide MDS patients into two major groups: high and low risk of AML progression. Recently, the evidence of a clinical correlation between the presence of a PI-PLC $\beta 1$ monoallelic gene deletion and the progression of MDS to AML opened new perspectives of research and treatments [Follo et al., 2008]. The gene encoding for PI-PLC $\beta 1$ has been mapped on chromosome 20p12. High-risk MDS patients should undergo allogenic hematopoietic stem cell transplantation (allo-HSCT), but for those who are not candidates, hypomethylating agents (HMAs), such AZA, are now the first therapeutic choice. HMAs can also be used in low risk MDS patients. AZA is both a hypomethylating and a direct cytotoxic agent for abnormal hematopoietic cells. Patients affected by MDS with a high risk of AML evolution show a reduction in the expression of the nuclear PI-PLC $\beta 1$ variant that can also be related to the presence of a mono-allelic deletion of the gene. As PIPLC $\beta 1$ b can physiologically regulate the progression through the G1 phase of the cell cycle, the drastic reduction of nuclear PI-PLC $\beta 1$ expression could alter the normal cell cycle in patients with MDS. These data could suggest the analysis of PI-PLC $\beta 1$ gene expression as a prognostic marker for MDS progression to AML. Nuclear PIPLC $\beta 1$ in MDS is also epigenetically relevant [Jhanwar, 2015]. In fact, several studies have shown that PI-PLC $\beta 1$ is a molecular target for AZA [Follo et al., 2009]. As a matter of fact, high-risk and lowrisk patients that respond to the drug have shown an early increase of nuclear PI-PLC $\beta 1$ expression, a reduction of PI-PLC $\beta 1$ promoter methylation, an induction of normal myeloid differentiation and a better prognosis. Moreover, as described above, the increase of PI-PLC $\beta 1$ expression and the reduction in PI-PLC $\beta 1$ promoter methylation are not only related to a favorable clinical response, but also to a durable response to the treatment. This effect resulted particularly interesting, because, as AZA treatment needed several cycles in order to observe a clinical response, the molecular response could specifically and rapidly predict the future patients' outcome during demethylating therapies. In this way it would be possible to personalize MDS patients' therapies in order to differentiate the ones who would benefit of the treatment from the ones who would be refractory to the treatment and thus could avoid it [Cocco et al., 2016]. Furthermore, several studies showed that in MDS high-risk patients there is an inverse correlation between PI-PLC $\beta 1$ and PI3K expression. PI3K can phosphorylate Protein Kinase B (PKB) also known as Akt [Toker and Marmiroli, 2014]. When there is a constitutive activation of Akt, through different phosphorylation, there is a decrease in MDS cell apoptosis that can be reverted after PI-PLC $\beta 1$ increase due to the demethylating therapies. Indeed PI-PLC $\beta 1$ increase could reduce the level of $\mathrm{p}$-Akt inducing a higher level of apoptosis [Follo et al., 2007]. This mechanism is still partially undiscovered, but a possible explication could be that as $\operatorname{PI}(4,5) \mathrm{P}_{2}$ is a common substrate of both PI-PLC $\beta 1$ and PI3Ks, the alterations in PI-PLC $\beta 1$ levels could cause $\mathrm{PI}(4,5) \mathrm{P}_{2}$ hydrolysis, reducing its availability for PI3K. This could eventually inhibit Akt activation [Follo et al., 2015]. All these mechanisms are extremely interesting for the potentiality of nuclear PI-PLC $\beta 1$ to become a prognosis stratification marker and a treatment predictive outcome marker in patients with MDS.

Nuclear inositides have been studied also in AML that can be a direct evolution of MDS. As we saw earlier in this prospect PIP4K2A catalyzes the phosphorylation of PI5P, synthetizing PI $(4,5) \mathrm{P}_{2}$. The role of PIP4K2A has been examined in human MLL-AF9 and in murine AML cells, and further investigated also in human AML samples [Jude et al., 2015]. AML cells were sensible to PIP4K2A depletion determining a cyclin-dependent kinase inhibitor CDKN1A (p21) accumulation, with thus an activation of the apoptosis. This mechanism seems to be related to the activation of mammalian Target of Rapamycin (mTOR), too. Considering this pathway it is then possible that PIP4K2A could also regulate mTOR. In addition it has been observed significant increase of PI5P following PIP4K2A depletion [Jude et al., 2015]. PI5P has been related to p53 and Akt activation and to cellular reactive oxygen species (ROS) accumulation in normal hematopoietic cells [Jones et al., 2013]. Moreover, PI5P might increase in response to several stress stimuli, mimicking cell stress induction. This mechanism could induce AML cell apoptosis or perhaps increase their susceptibility to other critical processes, such as DNA damage. It is still not clear why normal hematopoietic cells and AML cells have different response to PIP4K2A depletion, but these differences could be used for developing new therapeutic strategies against AML [Jude et al., 2015].

\section{MYOTONIC DYSTROPHIES (DM)}

DM are autosomal dominant neuromuscular degenerative disorders, characterized by a widely variable clinical picture and a slow progressive course, whose onset can occur at any age. The clinical picture is characterized by loss of muscle mass, mytonia, cataracts, defects in the cardiac conduction system, endocrine abnormalities, and cognitive deficits in congenital cases. Moreover, there is an anticipation phenomenon thus the disease onset tends to occur at an increasingly younger age from generation to generation in the same family.

These diseases are classified into DM type I (DM1) and DM type II (DM2). The majority of patients suffer of DM1, but both DM are caused by DNA tandem repeats that result in aberrant RNA 
accumulation in the nucleus that causes alteration in RNA-binding protein localization. In DM1 the mutated gene is called myotonic dystrophy protein kinase (DMPK) and encodes a myosin kinase expressed in skeletal muscles. This gene is located in the long arm of chromosome 19 (19q13.3). In the 3'UTR of this locus there is an unstable CTG triplet that can be repeated from 50 to 4000 times when in the normal population the range varies from 5 to 37 times. In DM2 there is a defect in zinc finger protein 9 (ZNF9) gene on chromosome 3 (3q21). The alteration is caused by CCTG expansion from 75 to over 11,000 , but in this case there seems to be no difference in the severity or the early onset of the disease [Cho and Tapscott, 2007]. CUG triplet repeat RNA-binding protein 1 (CUGBP1) plays a central role in alternative splicing of specific target genes and can interact with Initiation Factor 2 (eIF2) and Cyclin D3 inducing normal myogenic differentiation. This process can be altered in DM, indeed CUBBP 1-eIF2 interactions are reduced in myotonic dystrophy differentiating cells with an impaired muscle differentiation [Faenza et al., 2012]. Nevertheless, in DM1, ectopic expression of Cyclin D3 helps the increase of CUBBP1-eIF2 complex, improving the myogenic differentiation marker expression [Salisbury et al., 2008]. Moreover, during the myogenesis in DM cells there is a decrease of PI-PLC $\beta 1$ expression that could be related to the reduction in Cyclin D3 transcription and induction as described above in the role of PIPLC $\beta 1$ and Cyclin D3 in myogenic differentiation. Interestingly the normalization of PI-PLC $\beta 1$ expression in DM1 and DM2 myoblasts can cause a Cyclin D3 expression increase, determining a partially restored phenotype of the myotubes [Faenza et al., 2012]. All these data suggest that both PI-PLC $\beta 1$ and Cyclin D3 could be investigated for future possible molecular therapies in order to induce a correct skeletal muscle differentiation in DM.

\section{BRAIN DISORDERS}

PI-PLC isozymes are highly expressed in different brain structures. Specific neurotransmitters (NTs) and hormones trigger PI-PLC pathways, determining various activities in many brain regions [Yang et al., 2016]. PI-PLC $\beta 1$ is present at high concentration in hippocampus, amygdala, lateral septum, olfactory bulb, and cerebral cortex [Fukaya et al., 2008]. Indeed it has been related to cortical development and synaptic plasticity [Spires et al., 2005]. This PI-PLC has been associated with epilepsy and to schizophrenia in PI-PLC $\beta 1$ knocked out mice [Yang et al., 2016]. Losses of PI-PLC $\beta 1$ were also observed in patients with epileptic encephalopathies, schizophrenia and bipolar disorders [Kurian et al., 2010; Lo Vasco et al., 2012, 2013]. Also PI-PLC $\gamma 1$ has been correlated to neuronal cell migration and synaptic plasticity and its activity has been suggest to be of some relevance in epilepsy, Huntington disease, depression, bipolar disorders and Alzheimer disease [Yang et al., 2016]. Moreover, PIPLC $\beta 1$ seems to be central in the control of endocannabinoid neuronal excitability in most of the brain areas, indeed in the postsynaptic sites, and it is pivotal in the synthesis of DAG which hydrolysis catalyzed by DAG-Lipase $\alpha$ (DAGL $\alpha$ ) leads to 2-arachidonyl-glycerol (2-AG), the most represented CB1 cannabinoid receptor endogenous agonist. Released from the postsynaptic neuron, 2-AG can activate CB1 cannabinoid receptors on the presynaptic sites repressing other NT release [Montaña et al., 2012]. Moreover, PI-PLC $\beta 1$ can respond to two separate signals: depolarization and receptor activation, maintaining the brain inhibitory circuits through 2-AG [Del Caño et al., 2014]. Interestingly the pathway related to PI-PLC $\beta 1 / D A G L \alpha /$ $2-\mathrm{AG}$ seems to be at neuronal nuclear level. In adult rat brain, double immunofluorescence staining and confocal laser scanning showed an overlapping pattern of both PI-PLC $\beta 1$ and DAGL $\alpha$ with nuclear speckles marker SC-35 and NeuN/Fox3. In addition PI-PLC $\beta 1$ was also highly expressed in neuronal nuclear regions full of $\mathrm{PI}(4,5) \mathrm{P}_{2}$. These date were enforced by the fact that the co-localization with Nuclear Pore Complex and Lamin B1 was instead not found [Montaña et al., 2012]. There are also evidences of the relation between 2-AG and nuclear Peroxisome proliferator-activated receptor gamma (PPAR $\gamma$ ) as a receptor or as a prostaglandin precursor [Del Caño et al., 2014]. All these intriguing data prompt to better investigate the neuronal nuclear localization and mechanisms of PI-PLC $\beta 1$ related molecules in different pathophysiological brain functions. Furthermore, considering nuclear PI-PLC $\beta 1$ pivotal role in cell cycle regulation and the controversial and complicate role of cell cycle in several central nervous system insults and pathologies, it would be very fascinating to study nuclear PI-PLC $\beta 1$ functions during diverse brain injuries forms [Del Caño et al., 2014].

\section{INFERTILITY}

PI-PLC $\zeta$ is a specific sperm protein that has been related to nuclear infertility mechanisms. PI-PLC $\zeta$ activation and nuclear translocation mechanisms remain unknown, but at nuclear level this PI has been specifically connected with molecular oocyte activation. This PI-PLC is essential in inducing $\mathrm{Ca}^{2+}$ release via $\mathrm{IP}_{3}$ pathway [Saunders et al., 2002]. $\mathrm{Ca}^{2+}$ oscillations within the oocyte have been related to different processes responsible for its activation such as the cortical granule exocytosis, the release of meiotic arrest, the gene expression regulation, the recruitment of maternal mRNA, the pronuclear formation and the initiating of embryogenesis. The specific mechanism of these reactions is still unclear, but the central role of $\mathrm{Ca}^{2+}$ is fundamental for the beginning of embryogenesis [Amdani et al., 2016]. The principal cause of infertility after Intra Cytoplasmatic Sperm Injection (ICSI) is considered to be the alteration of oocyte activation mechanisms of which sperm defects seem to be the main responsible [Amdani et al., 2016]. From one side, cRNA PIPLC $\zeta$ and recombinant protein microinjections in mice and cattle oocyte determine $\mathrm{Ca}^{2+}$ oscillations and oocyte activation [Saunders et al., 2002]. From the other side infertile man sperm with alteration in PI-PLCל quantity and quality fail to induce $\mathrm{Ca}^{2+}$ oscillations and therefore oocyte activation [Amdani et al., 2016]. For these reasons it is possible that PIPLC $\zeta$ could be pivotal in fertiliy processes and could be considered a prognostic/diagnostic molecular marker in order to identify male patients that could benefit of assisted reproducive technology. Moreover, even if $\mathrm{Ca}^{2+}$ ionophores are the main agent for artificial oocyte activation, PIPLC $\zeta$ could be a safer potential therapeutic agent [Amdani et al., 2016].

\section{CONCLUSIONS}

In this prospect we tried to highlight our understanding of nuclear inositide signaling in different cell systems and pathologies. This topic is at the same time very testing and intriguing due to the technical difficulties in focusing on the nuclear localization of such complicate pathways and to the importance of these enzymes in several 
pathophysiological mechanisms. We analyzed the PI nuclear signaling through PI-PLCs mainly focusing on PI-PLC $\beta 1$, the most studied nuclear PI-PLC, and its related molecules like PI $(4,5) \mathrm{P}_{2}$, DAG, PKCs, Cyclin D3, and many others. We started from the beginning of this lipid nuclear study in the late 80s up to the most recent molecular and clinical implications and possibilities. PI-PLC $\beta 1$ and its associated molecules have been implicated to be essential in the cell cycle during both $\mathrm{G} 1 / \mathrm{S}$ transition and $\mathrm{G} 2 / \mathrm{M}$ progression. Moreover, PI-PLC $\beta 1$ is involved as pivotal enzyme in the normal mesenchymal cell differentiation in osteogenesis, myogenesis and adipogenesis. During the myogenic differentiation of $\mathrm{C} 2 \mathrm{C} 12$ cells it has been shown an increase of both nuclear PI-PLC $\beta 1$ and Cyclin D3. On the contrary, PI-PLC $\beta 1$ and Cyclin D3 reduction has been observed in DM, suggesting a key role of these enzymes in DM physiopathology. Similarly PI-PLC $\beta 1$ expression increases in the normal osteogenesis induced by BMP-2 in C2C12 cells and the down-regulation of PIPLC $\beta 1$ gene expression determines an inhibition of the osteogenic differentiation as demonstrated by ALP reduction. PI-PLC $\beta 1$ seems to increase also in adipogenesis and its inhibition seems to stop the increase of the Cyclin D3-CDK4 complex. This central role has been also demonstrated in the hematopoietic differentiation with probably opposite behavior between erythroid and myeloid differentiation. Very interestingly the nuclear inositide signaling via PI-PLC $\beta 1$ helped to create a bridge between molecular and clinical analyses, giving the opportunity to explore new potential therapeutic strategies and markers. Indeed there have been many progresses especially in understanding MDS pathological mechanism in AML evolution and in response to the therapies. Indeed nuclear PI-PLC $\beta 1$ could be used both as a prognostic marker in AML evolution and as a therapy predictive marker in MDS patients. PI-PLC $\beta 1$ has been investigated in the central nervous system where it seems to be involved in different processes and pathways in both brain cortex development and synaptic plasticity related to different disease. Nuclear PI-PLC $\beta 1$ is also involved in neurotransmitters regulation, suggesting a pivotal role in different brain mechanisms. Another PI-PLC isozyme that could be related to nuclear activities is PI-PLC $\zeta$ that is involved in oocyte activation and infertility processes where it could be considered as a molecular marker in order to identify male patients that could benefit of assisted reproducive technology. There are certainly many questions that remain to be clarified in relation to nuclear PI cycle and functions, but the importance of these themes and the related clinical prospects stimulate to persist on this field of investigation.

\section{ACKNOWLEDGMENTS}

This work was supported by grant from Intesa San Paolo to LC, grants from Italian MIUR-PRIN 2015 to LM and MYF, grant \#111104 from East Carolina University to JAM, grant from National Research foundation of Korea to PGS.

\section{REFERENCES}

Amdani SN, Yeste M, Jones C, Coward K. 2016. Phospholipase C zeta (PLCל) and male infertility: Clinical update and topical developments. Adv Biol Regul 61:58-67.
Bahk YY, Song H, Baek SH, Park BY, Kim H, Ryu SH, Suh PG. 1998. Localization of two forms of phospholipase $\mathrm{C}-\beta 1$, $\mathrm{a}$ and $\mathrm{b}$, in $\mathrm{C} 6 \mathrm{Bu}-1$ cells. Biochim Biophys Acta 1389(1):76-80.

Balla A, Balla T. 2006. Phosphatidy linositol 4-kinases: Old enzymes with emerging functions. Trends Cell Biol 16(7):351-361.

Boronenkov IV, Loijens JC, Umeda M, Anderson RA. 1998. Phosphoinositide signaling pathways in nuclei are associated with nuclear speckles containing pre-mRNA processing factors. Mol Biol Cell 9:3547-3560.

Bulley SJ, Clarke JH, Droubi A, Giudici ML, Irvine RF. 2015. Exploring phosphatidylinositol 5-phosphate 4-kinase function. Adv Biol Regul 57:193-202.

Bultsma Y, Keune W-J, Divecha N. 2010. PIP4Kbeta interacts with and modulates nuclear localization of the high-activity PtdIns5P-4-kinase isoform PIP4Kalpha. Biochem J 430(2):223-235.

Cho DH, Tapscott SJ. 2007. Myotonic dystrophy: Emerging mechanisms for DM1 and DM2. Biochim Biophys Acta 1772(2):195-204.

Cocco L, Finelli C, Mongiorgi S, Clissa C, Russo D, Bosi C, Quaranta M, Malagola M, Parisi S, Stanzani M, Ramazzotti G, Mariani GA, Billi AM, Manzoli L, Follo MY. 2015. An increased expression of PI-PLC 1 is associated with myeloid differentiation and a longer response to azacitidine in myelodysplastic syndromes. J Leukoc Biol 98(5):769-780.

Cocco L, Gilmour RS, Ognibene A, Letcher AJ, Manzoli FA, Irvine RF. 1987. Synthesis of polyphosphoinositides in nuclei of Friend cells. Evidence for polyphosphoinositide metabolism inside the nucleus which changes with cell differentiation. Biochem J 248(3):765-770.

Cocco L, Manzoli L, Faenza I, Ramazzotti G, Yang YR, McCubrey JA, Suh P-G, Follo MY. 2016. Modulation of nuclear PI-PLCbeta1 during cell differentiation. Adv Biol Regul 60:1-5.

Cocco L, Martelli AM, Gilmour RS, Ognibene A, Manzoli FA, Irvine RF. 1988. Rapid changes in phospholipid metabolism in the nuclei of Swiss 3T3 cells induced by treatment of the cells with insulin-like growth factor I. Biochem Biophys Res Commun 154(3):1266-1272.

Deacon EM, Pettitt TR, Webb P, Cross T, Chahal H, Wakelam MJO, Lord JM. 2002. Generation of diacylglycerol molecular species through the cell cycle: A role for 1-stearoyl, 2-arachidonyl glycerol in the activation of nuclear protein kinase C-betaII at G2/M. J Cell Sci 115(Pt5):983-989.

Del Caño G, Montaña M, Aretxabala X, González-Burguera I, López de Jesús M, Barrondo S, Sallés J. 2014. Nuclear phospholipase C- $\beta 1$ and diacylglycerol LIPASE- $\alpha$ in brain cortical neurons. Adv Biol Regul 54:12-23.

Divecha N, Banfic H, Irvine RF, Banfić H, Irvine RF. 1991. The polyphosphoinositide cycle exists in the nuclei of Swiss 3T3 cells under the control of a receptor (for IGF-I) in the plasma membrane, and stimulation of the cycle increases nuclear diacylglycerol and apparently induces translocation of protein kinase. Embo J 10(11):3207-3214.

Divecha N, Roefs M, Los A, Halstead J, Bannister A, D’Santos C. 2002. Type IPIPkinases interact with and are regulated by the retinoblastoma susceptibility gene product-pRB. Curr Biol 12(7):582-587.

Faenza I, Blalock W, Bavelloni S, Schoser B, Fiume R, Pacella S, Piazzi M, D'Angelo A, Cocco L. 2012. A role for PLC 1 in myotonic dystrophies type 1 and 2. FASEB J 26(7):3042-3048.

Feng Y, Cao J-H, Li X-Y, Zhao S-H. 2011. Inhibition of miR-214 expression represses proliferation and differentiation of C2C12 myoblasts. Cell Biochem Funct 29(5):378-383.

Filì C, Malagol M, Follo MY, Finelli C, Iacobucci I, Martinelli G, Cattina F, Candoni A, Fanin R, Gobbi M, Bocchia M, Defına M, Spedini P, Skert C, Manzoli L, Cocco L, Russo D. 2013. Prospective phase II study on 5-days azacitidine for treatment of symptomatic and/or erythropoietin unresponsive patients with low/INT-1-risk myelodysplastic syndromes. Clin Cancer Res 19(12):3297-3308. 
Fiume R, Ramazzotti G, Teti G, Chiarini F, Faenza I, Mazzotti G, Billi AM Cocco L. 2009. Involvement of nuclear PLCbeta1 in lamin B1 phosphorylation and $\mathrm{G} 2 / \mathrm{M}$ cell cycle progression. FASEB J 23(3):957-966.

Follo MY, Bosi C, Finelli C, Fiume R, Faenza I, Ramazzotti G, Gaboardi GC, Manzoli L, Cocco L. 2006. Real-time PCR as a tool for quantitative analysis of PI-PLC $\beta 1$ gene expression in myelodysplastic syndrome. Int J Mol Med 18(2):267-271.

Follo MY, Finelli C, Clissa C, Mongiorgi S, Bosi C, Martinelli G, Baccarani M, Manzoli L, Martelli AM, Cocco L. 2008. Phosphoinositide-Phospholipase C 1 mono-Allelic deletion is associated with myelodysplastic syndromes evolution into acute myeloid leukemia. J Clin Oncol 27(5):782-790.

Follo MY, Finelli C, Mongiorgi S, Clissa C, Bosi C, Testoni N, Chiarini F, Ramazzotti G, Baccarani M, Martelli AM, Manzoli L, Martinelli G, Cocco L. 2009. Reduction of phosphoinositide-phospholipase $C$ beta1 methylation predicts the responsiveness to azacitidine in high-risk MDS. Proc Natl Acad Sci USA 106(39):16811-16816.

Follo MY, Manzoli L, Poli A, McCubrey JA, Cocco L. 2015. PLC and PI3K/Akt/ mTOR signalling in disease and cancer. Adv Biol Regul 57:10-16.

Follo MY, Mongiorgi S, Bosi C, Cappellini A, Finelli C, Chiarini F, Papa V, Libra M, Martinelli G, Cocco L, Martelli AM. 2007. The Akt/mammalian target of rapamycin signal transduction pathway is activated in high-risk myelodysplastic syndromes and influences cell survival and proliferation. Cancer Res 67(9):4287-4294.

Follo MY, Mongiorgi S, Clissa C, Paolini S, Martinelli G, Martelli AM, Fioravanti G, Manzoli L, Finelli C, Cocco L. 2012. Activation of nuclear inositide signalling pathways during erythropoietin therapy in low-risk MDS patients. Leukemia 26(12):2474-2482.

Fukaya M, Uchigashima M, Nomura S, Hasegawa Y, Kikuchi H, Watanabe M. 2008. Predominant expression of phospholipase Cbeta1 in telencephalic principal neurons and cerebellar interneurons, and its close association with related signaling molecules in somatodendritic neuronal elements. Eur J Neurosci 28(9):1744-1759.

Gresset A, Sondek J, Harden TK. 2012. The phospholipase C isozymes and their regulation. Sub Cell Biochem 58:61-94.

Irvine RF. 2003. Nuclear lipid signalling. Nat Rev Mol Cell Biol 4(5):349-361.

Jhanwar SC. 2015. Genetic and epigenetic pathways in myelodysplastic syndromes: A brief overview. Adv Biol Regul 58:28-37.

Jones DR, Foulger R, Keune W-J, Bultsma Y, Divecha N. 2013. PtdIns5P is an oxidative stress-induced second messenger that regulates $\mathrm{PKB}$ activation. FASEB J 27(4):1644-1656.

Jude JG, Spencer GJ, Huang X, Somerville TDD, Jones DR, Divecha N, Somervaille TCP. 2015. A targeted knockdown screen of genes coding for phosphoinositide modulators identifies PIP4K2A as required for acute myeloid leukemia cell proliferation and survival. Oncogene 34(10):1253-1262.

Katagiri T, Yamaguchi A, Komaki M, Abe E, Takahashi N, Ikeda T, Rosen V, Wozney JM, Fujisawa-Sehara A, Suda T. 1994. Bone morphogenetic protein2 converts the differentiation pathway of $\mathrm{C} 2 \mathrm{C} 12$ myoblasts into the osteoblast lineage. J Cell Biol 127(6I):1755-1766.

Kim CG, Park D, Rhee SG. 1996. The Role of carboxyl-terminal basic amino acids in G q alpha-dependent activation, particulate association, and nuclear localization of phospholipase C-beta1. The J Biol Chem 271(35):21187-21192.

Kurian MA, Meyer E, Vassallo G, Morgan NV, Prakash N, Pasha S, Hai NA, Shuib S, Rahman F, Wassmer E, Cross JH, O'Callaghan FJ, Osborne JP, Scheffer IE, Gissen P, Maher ER. 2010. Phospholipase C beta 1 deficiency is associated with early-onset epileptic encephalopathy. Brain 133(10):2964-2970.

Lo Vasco VR, Longo L, Polonia P. 2013. Phosphoinositide-specific Phospholipase $C \beta 1$ gene deletion in bipolar disorder affected patient. J Cell Commun Signal 7(1):25-29.
Lo Vasco V, Cardinale G, Polonia P. 2012. Deletion of PLCB1 gene in schizophrenia-affected patients. J Cellu Mol Med 16(4):844-851.

Lukinovic-Skudar V, Donlagic L, Banfíc H, Visnjic D. 2005. Nuclear phospholipase C-beta1b activation during $\mathrm{G} 2 / \mathrm{M}$ and late $\mathrm{G} 1$ phase in nocodazole-synchronized HL-60 cells. Biochim Biophys Acta 1733(23):148-156.

Martelli AM, Fiume R, Faenza I, Tabellini G, Evangelista C, Bortul R, Follo MY, Falà F, Cocco L. 2005. Nuclear phosphoinositide specific phospholipase C (PI-PLC)-beta 1: A central intermediary in nuclear lipid-dependent signal transduction. Histol Histopathol 20(4):1251-1260.

Martelli AM, Gilmour RS, Bertagnolo V, Neri LM, Manzoli L, Cocco L. 1992. Nuclear localization and signalling activity of phosphoinositidase $C$ beta in Swiss 3T3 cells. Nature 358(6383):242-245.

Martelli AM, Neri LM, Gilmour RS, Barker PJ, Huskisson NS, Manzoli FA, Cocco L. 1991. Temporal changes in intracellular distribution of protein kinase C in Swiss 3T3 cells during mitogenic stimulation with insulin-like growth factor I and bombesin: Translocation to the nucleus follows rapid changes in nuclear polyphosphoinositides. Biochem Biophys Res Commun 177(1):480-487.

Montaña M, García del Caño G, López de Jesús M, González-Burguera I, Echeazarra L, Barrondo S, Sallés J. 2012. Cellular neurochemical characterization and subcellular localization of phospholipase $C \beta 1$ in rat brain. Neuroscience 222:239-268.

0'Carroll SJ, Mitchell MD, Faenza I, Cocco L, Gilmour RS. 2009. Nuclear PLCbeta1 is required for 3T3-L1 adipocyte differentiation and regulates expression of the cyclin D3-cdk4 complex. Cell Signal 21(6):926-935.

Osborne SL, Thomas CL, Gschmeissner S, Schiavo G. 2001. Nuclear PtdIns $(4,5) \mathrm{P} 2$ assembles in a mitotically regulated particle involved in pre-mRNA splicing. J Cell Sci 114(Pt13):2501-2511.

Payrastre B, Nievers M, Boonstra J, Breton M, Verkleij AJ, Van Bergen en Henegouwen PM. 1992. A differential location of phosphoinositide kinases, diacylglycerol kinase, and phospholipase $\mathrm{C}$ in the nuclear matrix. J Biol Chem 267(8):5078-5084.

Piazzi M, Blalock WL, Bavelloni A, Faenza I, Raffıni M, Tagliavini F, Manzoli L, Cocco L. 2015. PI-PLC $\beta 1 \mathrm{~b}$ affects Akt activation, cyclin E expression, and caspase cleavage, promoting cell survival in pro-B-lymphoblastic cells exposed to oxidative stress. FASEB J 29(4):1383-1394.

Poli A, Billi AM, Mongiorgi S, Ratti S, Mccubrey JA, Suh PG, Cocco L, Ramazzotti G. 2016a. Nuclear phosphatidylinositol signaling: Focus on phosphatidylinositol phosphate kinases and phospholipases C. J Cell Physiol 231(8):1645-1655.

Poli A, Fiume R, Baldanzi G, Capello D, Ratti S, Gesi M, Manzoli L, Graziani A, Suh PG, Cocco L, Follo MY. 2016b. Nuclear localization of diacylglycerol kinase alpha in K562 cells is involved in cell cycle progression. J Cell Physiol DOI: $10.1002 / j c p .25642$

Poli A, Ramazzotti G, Matteucci A, Manzoli L, Lonetti A, Suh P-G, McCubrey JA, Cocco L. 2014. A novel DAG-dependent mechanism links PKCa and Cyclin B1 regulating cell cycle progression. Oncotarget 5(22):11526-11540.

Ramazzotti G, Faenza I, Fiume R, Billi AM, Manzoli L, Mongiorgi S, Ratti S, McCubrey JA, Suh PG, Cocco L, Follo MY. 2016. PLC- $\beta 1$ and cell differentiation: An insight into myogenesis and osteogenesis. Adv Biol Regul 5-9. DOI: 10.1016/j.jbior.2016.10.005

Salisbury E, Sakai K, Schoser B, Huichalaf C, Schneider-Gold C, Nguyen H, Wang GL, Albrecht JH, Timchenko LT. 2008. Ectopic expression of cyclin D3 corrects differentiation of DM1 myoblasts through activation of RNA CUGbinding protein, CUG BP1. Exp Cell Res 314(11-12):2266-2278.

Saunders CM, Larman MG, Parrington J, Cox LJ, Royse J, Blayney LM, Swann K, Lai FA. 2002. PLC zeta: A sperm-specific trigger of Ca $\left(2^{+}\right)$oscillations in eggs and embryo development. Development 129(15):3533-3544.

Shah ZH, Jones DR, Sommer L, Foulger R, Bultsma Y, D'Santos C, Divecha N. 2013. Nuclear phosphoinositides and their impact on nuclear functions. FEBS J 280(24):6295-6310. 
Spires TL, Molnár Z, Kind PC, Cordery PM, Upton AL, Blakemore C, Hannan AJ. 2005. Activity-dependent regulation of synapse and dendritic spine morphology in developing barrel cortex requires phospholipase C- $\beta 1$ signalling. Cerebral Cortex 15(4):385-393.

Sun B, Murray NR, Fields AP. 1997. A role for nuclear phosphatidylinositolspecific phospholipase $C$ in the G2/M phase transition. J Biol Chem 272 (0021-9258):26313-26317.

Toker A, Marmiroli S. 2014. Signaling specificity in the Akt pathway in biology and disease. Adv Biol Regul 55:28-38.

Tribble EK, Ivanova PT, Grabon A, Alb JG, Jr., Faenza I, Cocco L, Brown HA, Bankaitis VA. 2016. Quantitative profiling of the endonuclear glycerophospholipidome of murine embryonicfibroblasts. J Lipid Res 57(8):1492-1506.

Vann LR, Wooding FB, Irvine RF, Divecha N. 1997. Metabolism and possible compartmentalization of inositol lipids in isolated rat-liver nuclei. Biochem J 327(Pt2):569-576.

Xu A, Wang Y, Xu LY, Gilmour RS. 2001. Protein kinase c $\alpha$-mediated negative feedback regulation is responsible for the termination of insulin-like growth factor I- induced activation of nuclear phospholipase $\mathrm{C}$ beta 1 in swiss 3T3 cells. J Biol Chem 276(18):14980-14986.

Yang YR, Kang DS, Lee C, Seok H, Follo MY, Cocco L, Suh PG. 2016. Primary phospholipase C and brain disorders. Adv Biol Regul 61:80-85. 